\title{
Multi Drug Resistant Pseudomonas aeruginosa in a Health
}

\section{Care Setting in Alexandria}

\author{
Amani Abaza*
}

\begin{abstract}
The emergence of Multi drug resistant Pseudomonas aeruginosa (MDRPA) among intensive care unit (ICU) patients is increasingly recognized as a public health threat worldwide. This work aimed to study the occurrence of MDRPA among critically ill patients in a health care setting in Alexandria. During a 12 months period, different clinical samples (sputum, endotracheal aspirates, blood, urine, and pus) obtained from ICU patients were tested for the isolation and identification of Pseudomonas aeruginosa ( $P$. aeruginosa) strains; that were screened for their antimicrobial susceptibility patterns using single disc diffusion method. Identified MDRPA strains were further tested for their susceptibility to polymyxin $E$ (colistin), polymyxin $B$, and tigecycline. Polymerase chain reaction (PCR) assay was performed to detect VIM and IMP MBL genes. Of the 105 $P$. aeruginosa strains isolated from various clinical samples, $20(19 \%)$ were found to be MDRPA, of which $16(80 \%)$ were sensitive to each of colistin and polymyxin B, while only $5(25 \%)$ strains were sensitive to tigecycline. PCR assay revealed that $9(45 \%)$ strains possessed VIM MBL gene and none $(0 \%)$ harbored IMP MBL gene. The occurrence of MDRPA strains among critically ill patients in this study was noticeable; with colistin and polymyxin $B$ being effective upon the majority of identified MDRPA strains, and VIM MBL gene was found to be significantly harbored.
\end{abstract}

Keywords: Multidrug resistance Pseudomonas aeruginosa, metallo beta lactamases, polymyxins, colistin, tigecycline, VIM \& IMP MBL genes.

\section{INTRODUCTION}

Pseudomonas aeruginosa ( $P$. aeruginosa)

is a major hospital-associated pathogen; that

can cause a wide spectrum of severe

infections, most notably in immuno-

compromised patients or those hospitalized in

intensive care units (ICUs).(1)
It is responsible for $10-15 \%$ of the nosocomial infections worldwide. Often these infections are hard to treat due to the natural resistance of the species, as well as its remarkable ability of acquiring further mechanisms of resistance to multiple

*Microbiology Department, Alexandria University Students' Hospital, Egypt. 
groups of antimicrobial agents. ${ }^{(2,3)}$

The multi drug resistant $P$. aeruginosa (MDRPA) is on the rise and the increase in its incidence raises serious concerns. (4) A review of studies on MDR and 'pan-drug resistant' $P$. aeruginosa infections revealed considerably different definitions used in the literature, ranging from resistance to a single antibiotic agent/class to resistance to all tested antibiotics. In the majority of the published studies, multidrug resistance was defined as resistance to at least three drugs from a variety of antibiotic classes, mainly antipseudomonal penicillins, aminoglycosides, cephalosporins, carbapenems and fluoroquinolones. ${ }^{(5,6)}$

A national surveillance of 13,999 nonduplicate $P$. aeruginosa isolates from ICU patients showed that multidrug resistance increased significantly, from $4 \%$ in 1993 to $14 \%$ in $2002 .{ }^{(7)}$

$P$. aeruginosa is intrinsically resistant to many drugs and is able to become resistant to virtually any antimicrobial agent. Acquired mechanisms contributing to resistance in $P$. aeruginosa include $\beta$-lactamases, notably the extended-spectrum $\beta$-lactamases and the carbapenemases that hydrolyze most $\beta$ lactams, aminoglycoside-modifying enzymes, and 16S rRNA methylases that provide highlevel pan-aminoglycoside resistance. Resistance to carbapenems is often mediated by production of Metallo-Beta-Lactamases $(\mathrm{MBL})$; a class $\mathrm{B}$ type of beta-lactamases that is the most worrisome and require bivalent metal ions, usually zinc for their activity. ${ }^{(8)}$ Five MBL types, namely, the IMP, VIM, SPM, GIM, and SIM types of MBLs, have been identified; however, the IMP and VIM types are the most commonly detected MBLs worldwide. ${ }^{(9,10)}$

$P$. aeruginosa, producing MBLs, was first reported from Japan in 1991 and since then has been described from various parts of the world, including Asia, Europe, Australia, South America, and North America. (11,12) In some countries, $P$. aeruginosa possessing MBLs constitute nearly $20 \%$ of all nosocomial isolates. (10) In India, published reports 
indicated that the prevalence of MBLs ranged from 7-65 \%, with a study reporting 34\% occurrence. $(13,14)$ MDRPA isolates have been responsible for several outbreaks in tertiary centers in different parts of the world, and have been associated with failure of therapy with carbapenems, illustrating the need for proper infection control practices. ${ }^{(15}$, 16) In Egypt, an outbreak in an ICU of a university hospital over 14 weeks period has been reported; where 25 ICU patients developed infections by MDRPA. ${ }^{(17)}$

The emergence of Gram-negative bacteria resistant to most available antibiotics has led to the readministration of polymyxin $B$ and polymyxin E (colistin) as "salvage" therapy in critically ill patients. Recent studies demonstrated acceptable effectiveness and considerably less toxicity than reported in older studies of polymyxins. ${ }^{(18)}$

Colistin, an old antibiotic also known as polymyxin $E$, has attracted more interest because of its significant activity against multiresistant $P$. aeruginosa, Acinetobacter baumannii and Klebsiella pneumoniae, and the low resistance rates to it. Because its use as an anti-pseudomonal agent was displaced by the potentially less toxic aminoglycosides in 1970s, our knowledge of this drug is limited. It is likely that colistin would be an important antimicrobial option against multi-resistant Gram-negative bacteria, for some years to come. ${ }^{(19)}$

This work aimed at studying the occurrence of MDRPA among critically ill patients in a health care setting in Alexandria, Egypt. It included the isolation and identification of $P$. aeruginosa strains, testing their antimicrobial susceptibility patterns and identifying MDRPA strains, studying the susceptibility patterns of MDRPA strains to polymyxin $\mathrm{E}$ (colistin), polymyxin $\mathrm{B}$, and tigecycline and detecting VIM and IMP MBL genes using PCR assay.

\section{MATERIAL AND METHODS:}

This study was carried out during a 12 months period from January 2009 till the end of December 2009. Different clinical samples 
obtained from patients admitted to the 3 ICUs

(General, Coronary and Intermediate) of the

Alexandria University Students' Hospital were

tested for the isolation and identification of MDRPA.

\section{1] Isolation and Identification of \\ $P$. aeruginosa strains: ${ }^{(20)}$}

All collected clinical samples (bloodendotracheal aspirates-sputum-pus-urine) were cultured on Blood and MacConkey's agar plates, and incubated at $37^{\circ} \mathrm{C}$ aerobically for 24 hours. The strains were identified as $P$. aeruginosa by conventional methods. The isolates that were Gram negative bacilli, oxidase positive with large flat pigmented colonies and a sweet or grape like odour, feathered edge, opaque, $\beta$ hemolytic on blood agar plates, and non lactose fermenting on MacConkey's agar plates were suspected of being Pseudomonas spp. Those that were positive for: citrate utilization, motility, and growth at $42^{\circ} \mathrm{C}$, negative for: Indole, MR and VP tests, and on TSI they showed alkaline slant /unchanged butt with no $\mathrm{H}_{2} \mathrm{~S}$ production or gas formation, were considered as $P$. aeruginosa.

\section{2] Antimicrobial Susceptibility Testing:} Identified $P$. aeruginosa strains were screened for their antimicrobial susceptibility using single disc diffusion method described by Bauer et al. (21) The test was done on Mueller Hinton agar plates, using the selected antibiotic discs with various concentrations including piperacillin, piperacillin /tazobactam, ceftazidime, cefotaxime,ceftriaxone, cefazolin, cefalothin, cefoxitin, aztreonam, meropenem, imipenem, gentamicin, tobramycin, amikacin, amoxicillin/ clavulanic acid, ampicillin/sulbactam, ciprofloxacin, and levofloxacin (Oxoid). Inhibition zones were measured and susceptibility was interpreted as susceptible (S), Intermediate (I) and resistant $(R)$ according to standard tables published by CLSI. (22)

Isolated $P$. aeruginosa strains were 
considered multi drug resistant when they were found to be resistant to at least 3 of the following antibiotic classes: anti pseudomonal penicillins, cephalosporins, monobactams, carbapenems, aminoglycosides, and fluoroquinolones.

MDRPA strains were further tested for their susceptibility to polymyxin E (colistin), polymyxin B, and tigecycline using disc diffusion method. Inhibition zones were interpreted according to CLSI guidelines. (22) 3] MBL genes identification:

All 20 identified MDRPA strains were tested for VIM and IMP MBL genes using PCR assay as follows:

\section{a) DNA Extraction:}

Fresh Culture of the tested strains of $P$. aeruginosa and control strains were suspended in $500 \mu \mathrm{l}$ of saline and vortexed to get a uniform suspension.

The cells were lysed by heating them at $100^{\circ} \mathrm{C}$ for ten minutes, and cellular debris was removed by centrifugation at $8000 \mathrm{rpm}$ for five minutes. The supernatant was used as a source of template for amplification.

b) Amplification Reaction: (23)

Duplex PCR amplification for the simultaneous detection of IMP and VIM MBL genes were carried out using primers supplied by Bioneer Corporation (Table 1). Aliquots of $2 \mu \mathrm{l}$ DNA extracts were used for amplification in a $25-\mu \mathrm{l}$ PCR reaction mixture containing (DreamTaq ${ }^{\mathrm{TM}}$ Green PCR Master mix, Fermantas). Amplification was performed in a Biocycler Tc-S Thermocycler (Boeco Germany).The temperature profile used for the amplification was; $94^{\circ} \mathrm{C}$ for 2 min (initial denaturation), then 30 cycles of $94^{\circ} \mathrm{C}$ for 1 min (DNA denaturation), $54^{\circ} \mathrm{C}$ for $1 \mathrm{~min}$ (primer annealing) and $72^{\circ} \mathrm{C}$ for $1.5 \mathrm{~min}$ (primer extension) and a final extension step of $5 \mathrm{~min}$ at $72^{\circ} \mathrm{C}$. The amplification products were analyzed by agarose gel electrophoresis and 
ethidium bromide staining. The were distributed as $7(35 \%)$ from sputum amplification was performed in samples, 5(25\%) from pus, 3(15\%) from duplicates and controls were each of urine and endotracheal aspirates, included in each assay. and $2(10 \%)$ from blood samples (Table 2$)$.

\section{4] Statistical analysis:}

All identified MDRPA strains in this

- The results of the present study were tabulated and statistical analyses were conducted using PC with the software: Statistical Package for the Social Sciences (SPSS) version 15 and Excel.

- Statistical significance was set at 5\% $(P<0.05)$. The Z- test was done.

\section{RESULTS}

In a 12 months period, a total of 105 $P$. aeruginosa strains were isolated from different clinical samples collected from patients admitted to the ICUs of the Alexandria University Students' Hospital. Twenty $P$. aeruginosa strains (19\%) were found to be multi drug resistant to tested antimicrobial agents, of which 16 isolates $(80 \%)$ and $4(20 \%)$ were reported from males and females respectively. They study were resistant to all tested antimicrobial agents except for only 2 strains $(10 \%)$ that were susceptible to aztreonam and one strain (5\%) to meropenem.

Of the 20 tested MDRPA strains, 9 (45\%) strains were found to harbor VIM $M B L$ gene, while none of the tested strains (0\%) was positive for IMP MBL gene by PCR assay. The 9 VIM positive strains were recovered from 5 (55\%) females and $4(45 \%)$ males, where 5 out of $9(55 \%)$ strains were isolated from sputum samples and 4 (45\%) were from pus samples.

As regards the susceptibility patterns of the 20 MDRPA strains to colistin, polymyxin B and tigecycline; 16 (80\%) were sensitive to colistin and polymyxin $B$, 
while only 5 (25\%) strains were sensitive to tigecycline (Table 3).

\section{DISCUSSION}

The emergence of MDRPA in hospital acquired infections among ICU patients is increasingly recognized and is related to high morbidity and mortality. ${ }^{(4,23)}$ Its prevalence has increased over the past decade and has become a major public health concern. ${ }^{(7,24)}$ The phenomenon of multi drug resistant pathogens had emerged not only in Egypt but also worldwide due to the excessive antibiotic misuse. $(25,26)$

The present work was conducted to study the occurrence of MDRPA among critically ill patients in ICUs. Intensive care patients create an environment for infection because of the debilitating effect of prolonged hospitalization, serious underlying disease, and compromised membrane and skin barriers following the application of invasive medical equipment ( as airway, catheters, etc)(27)

Reported rates of MDRPA infection varied from $0.6-32 \%$ depending on geographic location and type of surveillance study.(28) In the present study, of the 105 isolated $P$. aeruginosa strains; 20 (19\%) were found to be MDRPA. Nearly similar results were reported in a university hospital in Italy (29), where MDRPA recorded a rate of 17\%, while in the United States (U.S.), Flamm et al recorded a rate of 23 to $26 \%$ of MDRPA isolates collected from 1999 to 2002. (30) In 2005-2007, Tam et al documented a prevalence rate of $10-17 \%$. $^{(31)}$

Rates of MDRPA in individual institutions are even higher than those reported in large surveillance studies. Earlier in a university hospital in Brazil, $10.2 \%$ of $P$. aeruginosa isolates (48/472) were reported as MDRPA in 1992. (32) An institution in Greece declared that $24.3 \%(25 / 103)$ of non-duplicate $P$. aeruginosa isolates from 1996-1997 were resistant to all antimicrobials tested. (33) A higher isolation rate of $32 \%(13 / 41)$ was reported in another academic medical center in the U.S. in 2002. (34) While in 2007, Lodise 
et al found that among $351 P$. aeruginosa infected patients, the proportion of MDRPA was $35 \%$. ${ }^{(24)}$

In this work, different clinical samples were collected and tested for MDRPA including blood, endotracheal aspirates, sputum, urine, and pus from wounds. The most common site of isolation of MDRPA was sputum 7 (35\%), followed by pus from wounds $5(25 \%)$, endotracheal aspirates and urine samples revealed $3(15 \%)$ each, while blood yielded only 2 (10\%) of MDRPA isolates (Table 2). In concordance to the present findings, in a study conducted in Egypt (2004) to identify the causative agent of an outbreak in an ICU of a university hospital, sputum yielded the highest rate of MDRPA isolation $14(38.8 \%) .(17)$ This could be attributed to the fact that most of the patients admitted to ICUs are mechanically ventilated and have respiratory infections. This agreed with Gales et al (2001) who documented that the respiratory tract was the most frequent source of $P$. aeruginosa isolates, followed by wounds, urine, and bloodstream. ${ }^{(6)}$

The increasing prevalence of MDRPA in ICU patients has rekindled interest in polymyxins, as a salvage therapy to these patients; where limited therapeutic options have forced infectious disease clinicians and microbiologists to reappraise the clinical application of polymyxin antibiotics discovered more than 50 years ago. (35) In the current study, the susceptibility pattern for polymyxin E (colistin), polymyxin B and tigecycline were tested. It was found that $16(80 \%)$ of isolated MDRPA were sensitive to colistin and polymyxin B (Table 3). In agreement with our findings, multiple studies have suggested that polymyxins are effective and safe therapy for infections caused by MDR strains. However, the role of these antibiotics has not been definitively established. Most studies have included only a small number of patients and the polymyxin was administered in combination with several other different antibiotics. $(36,37)$

$$
\text { Colistin (polymyxin E) remains active on }
$$


virtually all MDRPA isolates, and increasingly appears as the last available option to treat infections caused by these strains. However, the emergence of colistin resistance has been reported in $P$. aeruginosa, which may announce the spread of pan-resistant strains in a close future. ${ }^{(38)}$

On the other hand, tigecycline the new glycylcycline with an expanded broadspectrum antibiotic, including inhibition of Gram-positive, Gram-negative, atypical, anaerobic, and antibiotic-resistant organisms; has less activity against $\boldsymbol{P}$. aeruginosa than it does against some of the other nonEnterobacteriaceae; ${ }^{(39,40)}$ this is in accordance with the results of this study, where only 5 (25\%) strains were sensitive to tigecycline.

Carbapenemases are beta-lactamases with versatile hydrolytic capacities. They have the ability to hydrolyze penicillins, cephalosporins, monobactams, and carbapenems. Carbapenemases are members of the molecular class $A, B$, and $D$ beta-lactamases. Class $A$ and $D$ enzymes have a serine-based hydrolytic mechanism, while class B enzymes are MBLs that are the most worrisome of all betalactamases. ${ }^{(9)}$

MBL have been reported for $P$. aeruginosa isolates from nearly all regions of the globe. (41) VIM-type MBLs are predominant in Europe, particularly in the Mediterranean region. Recently, 18 VIM-type variants have been described and another 4 have been assigned to the database. ${ }^{(42)}$

In the present study, all the 20 identified MDRPA strains were tested for VIM and IMP MBL genes using PCR assay. Nine strains (45\%) were positive for VIM MBL gene, while none of the isolates $(0 \%)$ was positive for IPM MBL type, and they were found to be statistically significant (Table 1 \& figure1). This agrees with what was previously published by Manoharan et al (2010), where PCR testing detected VIM type of MBL among 15 of the 48 tested isolates (31\%); and all were negative for IMP MBL gene. (23) In Greece, Siarkou et al (2009) recorded that of 
29 non replicate $\quad P$. aeruginosa isolates resistant to carbapenems and ceftazidime, 14 (48\%) were positive for VIM MBL production.

(42) On the contrary, Ramos et al (2008) in Mexico declared that of the 40 tested carbapenem-resistant isolates with PCR for VIM and IMP MBL specific primers, only $13(32.5 \%)$ isolates displayed a MBL phenotype and yielded positive amplicons with the IMP MBL specific primers. ${ }^{(43)}$

Multiple antibiotic resistance in bacterial populations is a pervasive and growing clinical problem, which is recognized as a threat to public health. Hence, there is a need to conduct area-specific monitoring studies to profile different pathogens responsible for specific infections and their resistance patterns.

\section{Conclusions:}

1) The occurrence of MDRPA strains among critically ill patients was noticeable, where they represented 19\% of the isolated strains.

2) Colistin and polymyxin B were found to be active upon the majority of identified MDRPA strains, where $80 \%$ of the strains were sensitive to both of them, while tigecycline was found to be less effective as only $25 \%$ of strains were sensitive to it.

3) VIM MBL gene was significantly present among the tested MDRPA strains, where 45\% were positive by PCR assay.

\section{RECOMMENDATION}

MDRPA health care associated infections are on the rise; hence rigorous studying and monitoring of these clinically significant strains especially among critically ill patients should be emphasized, so as to generate data that would help clinicians to choose the correct treatment.

\section{Acknowledgment:}

I would like to express my heartily thanks and sincere appreciation to Dr. Walaa Hazzah for her kind support and laborious effort during the performance of the PCR assay in this study. 
Table 1: Oligonucleotides used as primers for testing 20 MDRPA strains by Duplex PCR assay.

\begin{tabular}{|c|c|c|c|c|c|}
\hline \multirow[t]{2}{*}{ Primers } & \multirow[t]{2}{*}{ Sequence $\left(5^{\prime} \text { to } 3^{\prime}\right)^{(23)}$} & \multirow[t]{2}{*}{$\begin{array}{c}\text { Amplicon } \\
\text { (nucleotide) }\end{array}$} & \multicolumn{2}{|c|}{$\begin{array}{l}\text { Positive } \\
\text { strains }\end{array}$} & \multirow[t]{2}{*}{ Z test } \\
\hline & & & No & $\%$ & \\
\hline IMP-A & GAA GGY GTT TAT GTT CAT AC & 587-bp amplicon & $0 / 20$ & 0 & \multirow{4}{*}{$3.41^{*}$} \\
\hline IMP-B & GTA MGT TTC AAG AGT GAT GC & & & & \\
\hline VIM2004A & GTT TGG TCG CAT ATC GCA AC & 382-bp amplicon & $9 / 20$ & 45 & \\
\hline VIM2004B & AAT GCG CAG CAC CAG GAT AG & & & & \\
\hline
\end{tabular}

Table 2: Distribution of the 20 MDRPA strains isolated from different clinical samples, Alexandria.

\begin{tabular}{|l|c|c|}
\hline \multirow{2}{*}{ Type of Samples } & \multicolumn{2}{|c|}{ MDRPA } \\
\cline { 2 - 3 } & No & $\%$ \\
\hline Sputum & 7 & 35 \\
\hline Pus (purulent discharge) & 5 & 25 \\
\hline Urine & 3 & 15 \\
\hline Endotracheal aspirates & 3 & 15 \\
\hline Blood & 2 & 10 \\
\hline Total & 20 & 100 \\
\hline
\end{tabular}

Table 3: Susceptibility patterns of the 20 MDRPA to colistin, polymyxin B and tigecycline.

\begin{tabular}{|l|l|l|l|l|l|l|}
\hline \multirow{3}{*}{ Susceptibility patterns } & \multicolumn{2}{|l|}{ Susceptible } & \multicolumn{2}{l|}{ Resistant } & \multicolumn{2}{l|}{ Total } \\
\cline { 2 - 7 } Tested antimicrobials & No & $\%$ & No & $\%$ & No & $\%$ \\
\hline Colistin & 16 & 80 & 4 & 20 & 20 & 100 \\
\hline Polymyxin B & 16 & 80 & 4 & 20 & 20 & 100 \\
\hline Tigecycline & 5 & 25 & 15 & 75 & 20 & 100 \\
\hline
\end{tabular}




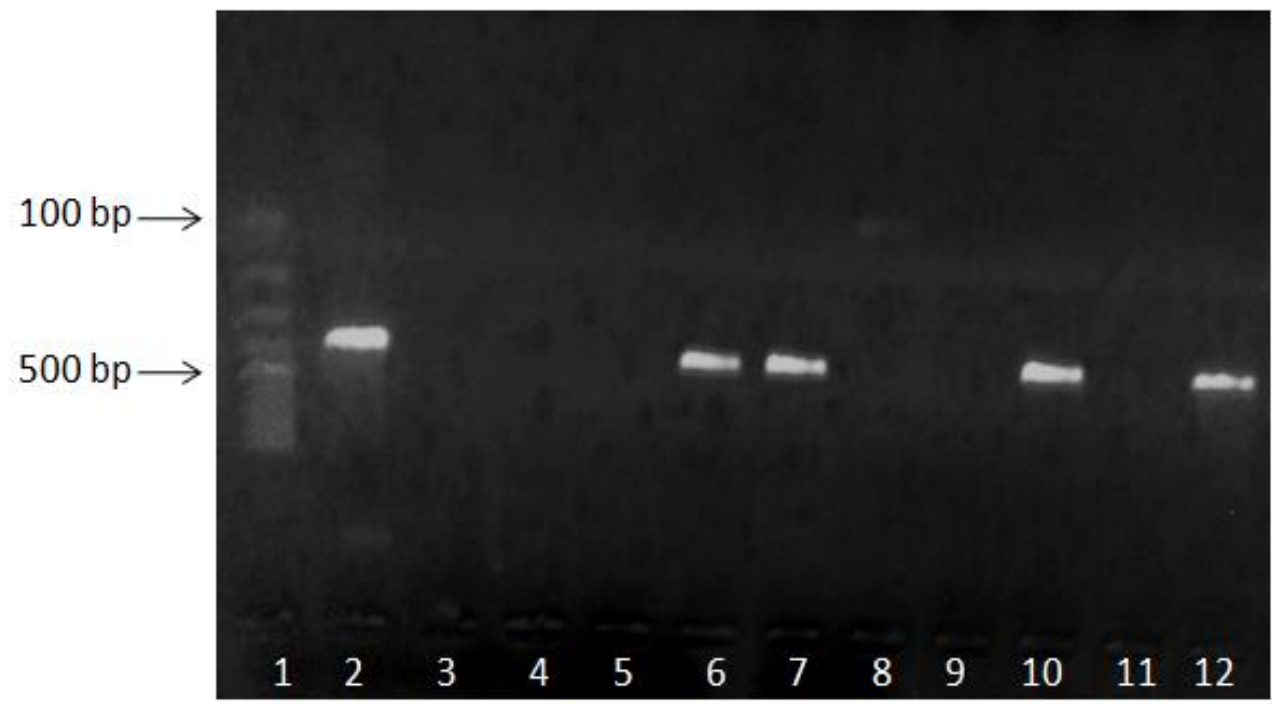

Figure 1: Duplex PCR of MDRPA strains with positive VIM MBL gene at 382-bp.

\section{REFERENCES}

1. Zavascki AP, Carvalhaes CG, Picão RC, Gales AC. Multidrug-resistant Pseudomonas aeruginosa and Acinetobacter baumannii: resistance mechanisms and implications for therapy. Expert Rev Anti Infect Ther 2010 Jan; 8(1):71-93.

2. Blanc DS, Petignat C, Janin B, Bille J, Francioli $P$. Frequency and molecular diversity of Pseudomonas aeruginosa upon admission and during hospitalization: a prospective epidemiologic study. Clin Microbiol Infect 1998; 4: 242-7.

3. Strateva T, Yordanov D. Pseudomonas aeruginosa - a phenomenon of bacterial resistance. J med microbial 2009; 58 (9):1133-48.

4. Gupta V. Metallo beta lactamases in Pseudomonas aeruginosa and Acinetobacter species. Expert Opin Investig Drugs 2008; 17(2):131-43.
5. Falagas ME, Koletsi PK, Bliziotis IA. The diversity of definitions of multidrugresistant (MDR) and pandrug-resistant (PDR) acinetobacter baumannii and Pseudomonas aeruginosa. J Med Microbiol 2006; 55(Pt 12):1619-29.

6. Gales AC, Jones RN, Turnidge J, Rennie R, Ramphal R. Characterization of Pseudomonas aeruginosa isolates: occurrence rates, antimicrobial susceptibility patterns, and molecular typing in the global SENTRY Antimicrobial Surveillance Program, 1997-1999. Clin Infect Dis 2001; 32(Suppl. 2): S146-55.

7. Obritsch MD, Fish DN, MacLaren R, Jung $R$. National surveillance of antimicrobial resistance in Pseudomonas aeruginosa isolates obtained from intensive care unit patients from 1993 to 2002. Antimicrob Agents Chemother 2004; 48:4606-10. 
8. Bush K. í-Lactamases of increasing clinical importance. Curr Pharm Des 1999; 5:839-45.

9. Queenan AM, Bush K. Carbapenemases: the versatile betalactamases. Clin Microbiol Rev 2007; 20:440-58.

10. Walsh TR, Toleman MA, Poirel L, Nordmann P. Metallo-beta-lactamases: the quiet before the storm? Clin Microbiol Rev 2005; 18:306-25.

11. Gales AC, Menezes LC, Silbert S, Sader HS. Dissemination in distinct Brazilian regions of an epidemic carbapenemresistant Pseudomonas aeruginosa producing SPM Metallo-ílactamase. J Antimicrob Chemother 2003;52:699-702.

12. Lee K, Lee WG, Uh Y, Ha GY, Cho J, Chong Y. VIM- and IMP-type metallo-ílactamase-producing Pseudomonas spp. and Acinetobacter spp. in Korean hospitals. Emerg Infect Dis 2003;9:86871.

13. Behera B, Mathur $P$, Das A, Kapil A, Sharma V. An evaluation of four different phenotypic techniques for detection of metallo- $\beta$-lactamase producing Pseudomonas aeruginosa. Ind J Med Microbiol 2008;26:233-7.

14. Castanheira M, Bell JM, Turnidge JD, Mathai D, Jones RN. Carbapenem Resistance among Pseudomonas aeruginosa Strains from India: Evidence for Nationwide Endemicity of Multiple Metallo- $\beta$-Lactamase Clones (VIM-2, -5, -6 , and -11 and the Newly Characterized VIM-18). Antimicrob Agents Chemother 2009; 53:1225-7.

15. Cornaglia G, Mazzariol A, Lauretti L, Rossolini GM, Fontana R. Hospital outbreak of carbapenem-resistant Pseudomonas aeruginosa producing VIM-1, a novel transferable metallo- $\beta-$ lactamase. Clin Infect Dis 2000; 31:111925.

16. Crespo MP, Woodford N, Sinclair A, Kaufmann ME, Turton J,Glover J,Velez
JD, Castaneda CR, Recalde M, Livermore DM. Outbreak of carbapenemresistant Pseudomonas aeruginosa producing VIM-8, a novel metallo-ßlactamase, in a tertiary care center in Cali, Colombia. J Clin Microbiol 2004; 42:5094-101.

17. Khamis N. Beta-Lactamase and Mex-R Gene Mutation in a Nosocomial Outbreak of Carbapenem - Resistant Pseudomonas. Egypt J Med Lab Sci 2004; 13(1).

18. Michalopoulos A, Falagas ME. Colistin and polymyxin $B$ in critical care. Crit Care Clin 2008 Apr; 24(2):377-91.

19. Li J, Nation RL, Milne RW, Turnidge JD, Coulthard K. Evaluation of colistin as an agent against multi-resistant Gramnegative bacteria. Int $\mathrm{J}$ Antimicrob Agents 2005 Jan; 25(1):11-25.

20. Forbes BA, Sahm DF, Weissfeld AS. Bailey and Scott's Diagnostic Microbiology. 10th ed. St Louis: Mosby 1998 (bailey scott).

21. Bauer AW, Kirby WMM, Sherris JC. Antibiotic susceptibility testing by standardized single disk diffusion method. Am J Clin Path1966; 45: 493-6.

22. Clinical and Laboratory Standards Institute/ CLSI. Performance standards for antimicrobial susceptibility testing; Twentieth international supplement M100-S20.January 2010. Wayne, PA, USA.

23. Manoharan A, Chatterjee S, Mathai D, SARI Study Group. Detection and characterization of metallo beta lactamases producing Pseudomonas aeruginosa. Ind $\mathrm{J}$ Med Microbiol 2010; 28 (3): 241-4.

24. Lodise TP, Miller CD, Graves J, Furuno JP, McGregor JC, Lomaestro B, Graffunder E, McNutt L. Clinical Prediction Tool To Identify Patients with Pseudomonas aeruginosa Respiratory Tract Infections at Greatest Risk for Multidrug Resistance. Antimicrob Agents Chemother 2007; 51(2): 417-22. 
25. El Kholy A, Baseem H, Hall GS, Procop GW, Longworth DL: Antimicrobial resistance in Cairo, Egypt 1999-2000: a survey of five hospitals. J Antimicrob Chemother 2003; 51:625-30.

26. Jean SS, Teng LJ, Hsueh PR, Ho SW, Luh KT: Antimicrobial susceptibilities among clinical isolates of extendedspectrum cephalosporin-resistant Gramnegative bacteria in a Taiwanese University Hospital. J Antimicrob Chemother 2002; 49:69-76.

27. Savas L, Duran N , Savas N, Onlen Y, Ocak S. The Prevalence and Resistance Patterns of Pseudomonas aeruginosa in Intensive Care Units in a University Hospital. Turk J Med Sci 2005; 35: 31722.

28. Obritsch MD, Fish DN, MacLaren R, Jung R. Nosocomial Infections Due to Multidrug-Resistant Pseudomonas aeruginosa: Epidemiology and Treatment Options. Pharmacotherapy2005; 25(10):1353-64.

29. Tacconelli E, Tumbarello M, Bertagnolio $S$, et al. Multidrug-resistant Pseudomonas aeruginosa bloodstream infections: analysis of trends in prevalence and epidemiology. Emerg Infect Dis 2002; 8:220-1.

30. Flamm R K, Weaver MK, Thornsberry C, Jones ME, Karlowsky JA, Sahm DF. Factors associated with relative rates of antibiotic resistance in Pseudomonas aeruginosa isolates tested in clinical laboratories in the United States from 1999 to 2002. Antimicrob Agents Chemother 2004; 48:2431-6.

31. Tam VH, Chang KT, Abdelraouf $\mathrm{K}$, et al. Prevalence, resistance mechanisms, and susceptibility of multidrug-resistant bloodstream isolates of Pseudomonas aeruginosa. Antimicrob Agents Chemother 2010; 54(3):1160-4.

32. Arruda EAG, Marinho IS, Boulos M, et al. Nosocomial infections caused by multiresistant Pseudomonas aeruginosa.
Infect Control Hosp Epidemiol 1999;20:620-3.

33. Sofianou D, Tsakris A, Skoura L, Douboyas J. Extended high-level crossresistance to antipseudomonal antibiotics amongst Pseudomonas aeruginosa isolates in a university hospital. $\mathrm{J}$ Antimicrob Chemother 1997; 40:740-2.

34. Jung R, Fish DN, Obritsch MD, et al. Prevalence of multidrug resistant Pseudomonas aeruginosa in an urban tertiary-care teaching hospital. J Hosp Infect 2004; 57:105-11.

35. Li J, Nation RL, Turnidge JD, Milne RW, Coulthard K, Rayner CR, Paterson DL. Colistin: there-emerging antibiotic for multidrug-resistant Gram-negative bacterial infections. Lancet Infect Dis 2006 Sep; 6(9):589-601.

36. Sobieszczyk ME, Furuya EY, Hay CM, et al. Combination therapy with polymyxin $B$ for the treatment of multidrug-resistant gram-negative respiratory tract infections. J Antimicrob Chemother 2004; 54:566-9.

37. Tascini C,Gemingnani G,Ferranti S, et al. Microbiological activity and clinical efficacy of colistin and rifampin combination in multidrug-resistant Pseudomonas aeruginosa infection. J Chemother 2004;16:282-7.

38. Michalopoulos A, Falagas ME. Colistin and Polymyxin B in Critical Care. Crit Care Clin 2008; 24: 377-91.

39. Rose WE, Rybak MJ.Tigecycline: first of a new class of antimicrobial agents. Pharmacotherapy 2006 Aug; 26(8):1099110.

40. Bradford PA. In Vitro Activity of Tigecycline against Isolates from Patients Enrolled in Phase 3 Clinical Trials of Treatment for Complicated Skin and Skin-Structure Infections and Complicated Intra-Abdominal Infections. Clin Infect Dis 2005;41(Suppl5):S315-32.

41. Lister PD, Wolter DJ, Hanson ND.

Antibacterial-Resistant Pseudomonas aeruginosa: Clinical Impact and Complex 
Regulation of Chromosomally Encoded Resistance Mechanisms. Clin Microbiol Rev 2009; 22(4): 582-610.

42. Siarkou VI, Vitti D, Protonotariou E, Ikonomidis A, Sofianou D. Molecular Epidemiology of Outbreak-Related Pseudomonas aeruginosa Strains Carrying the Novel Variant blaVIM-17 Metallo- $\beta$-Lactamase Gene. Antimicrob Agents Chemother 2009; 53(4): 1325-30.
43. Ramos UG, Morfin-Otero R, Sader HS, Jones RN, Hernández, RodriguezNoriega E, Sanchez A, Carrillo B, Esparza-Ahumada S, Silva-Sanchez. Metallo- $\beta$-Lactamase Gene blalMP-15 in a Class 1 Integron, In95, from Pseudomonas aeruginosa Clinical Isolates from a Hospital in Mexico. Antimicrob Agents Chemother 2008; 52(8): 2943-6. 